\title{
On Asymptotic Distribution of Parameter Free tests for Ergodic Diffusion Processes
}

\author{
Yury A. Kutoyants \\ Laboratoire de Statistique et Processus, Université du Maine \\ 72085 Le Mans, Cédex 9, France
}

\begin{abstract}
We consider two problems of constructing of goodness of fit tests for ergodic diffusion processes. The first one is concerned with a composite basic hypothesis for a parametric class of diffusion processes, which includes the Ornstein-Uhlenbeck and simple switching processes. In this case we propose asymptotically parameter free tests of Cramér-von Mises type. The basic hypothesis in the second problem is simple and we propose asymptotically distribution free tests for a wider class of trend coefficients.
\end{abstract}

MSC 2000 Classification: 62M02, 62G10, 62G20.

Key words: Cramér-von Mises tests, ergodic diffusion process, goodness of fit test, asymptotically distribution free.

\section{Introduction}

In this paper we consider two different goodness of fit (GoF) hypotheses testing problems for the diffusion process

$$
\mathrm{d} X_{t}=S\left(X_{t}\right) \mathrm{d} t+\sigma\left(X_{t}\right) \mathrm{d} W_{t}, \quad X_{0}, \quad 0 \leq t \leq T .
$$

In the first problem the observed process under the basic hypothesis $\left(\mathcal{H}_{0}\right)$ satisfies the stochastic differential equation

$$
\mathrm{d} X_{t}=-\beta \operatorname{sgn}\left(X_{t}-\alpha\right)\left|X_{t}-\alpha\right|^{\gamma} \mathrm{d} t+\sigma \mathrm{d} W_{t}, \quad X_{0}, \quad 0 \leq t \leq T,
$$

where $\vartheta=(\alpha, \beta) \in \Theta$ is the unknown parameter, $\beta>0, \gamma \geq 0$ and $\sigma>0$. Therefore the hypothesis is parametric composite. 
In the second problem we assume that under the basic hypothesis $\left(\mathcal{H}_{0}\right)$ the observed process satisfies

$$
\mathrm{d} X_{t}=S_{0}\left(X_{t}\right) \mathrm{d} t+\sigma\left(X_{t}\right) \mathrm{d} W_{t}, \quad X_{0}, \quad 0 \leq t \leq T,
$$

where $S_{0}(\cdot)$ is a known function, i.e., $\left(\mathcal{H}_{0}\right)$ is simple.

In both models the alternatives are nonparametric and, under the hypothesis $\mathcal{H}_{0}$, the diffusion processes are assumed to be ergodic with the invariant densities $f(\vartheta, x)$ and $f_{S_{0}}(x)$ respectively. We denote the corresponding distribution functions by $F(\vartheta, x)$ and $F_{S_{0}}(x)$.

Our goal is to construct the goodness of fit tests which provide the fixed limit error $\varepsilon \in(0,1)$. Introduce the class $\mathcal{K}_{\varepsilon}$ of such tests, i.e., the tests $\bar{\psi}_{T}$ satisfying the relations

$$
\lim _{T \rightarrow \infty} \mathbf{E}_{\vartheta} \bar{\psi}_{T}=\varepsilon \quad \text { for } \quad \text { all } \quad \vartheta \in \Theta
$$

and

$$
\lim _{T \rightarrow \infty} \mathbf{E}_{S_{0}} \bar{\psi}_{T}=\varepsilon
$$

in the first and the second problems respectively.

All tests studied in the present work are of the form $\hat{\psi}_{T}=\mathbb{I}_{\left\{\Delta_{T}>c_{\varepsilon}\right\}}$, where $\Delta_{T}$ is the Cramér-von Mises type statistic. More precisely, in the first problem $\Delta_{T}$ is either of the $L_{2}$ distances $D\left(\hat{F}_{T}(x), F\left(\hat{\vartheta}_{T}, x\right)\right)$ and $D\left(\hat{f}_{T}(x), f\left(\hat{\vartheta}_{T}, x\right)\right)$, where $\hat{F}_{T}(x)$ is the empirical distribution function, $\hat{f}_{T}(x)$ is the local time estimator of the invariant density and $\hat{\vartheta}_{T}$ is the maximum likelihood estimator (MLE) of the parameter $\vartheta$. Similarly, in the second problem $\Delta_{T}$ is one of the distances $D\left(\hat{F}_{T}(x), F_{S_{0}}(x)\right)$ and $D\left(\hat{f}_{T}(x), f_{S_{0}}(x)\right)$.

Let us denote by $\Delta(\vartheta)$ and $\Delta\left(S_{0}\right)$ the limits (in distribution) of the test statistics in the first and the second problems. Then the thresholds $c_{\varepsilon}$ in these tests have to satisfy the equations

$$
\mathbf{P}_{\vartheta}\left\{\Delta(\vartheta)>c_{\varepsilon}\right\}=\varepsilon, \quad \mathbf{P}_{S_{0}}\left\{\Delta\left(S_{0}\right)>c_{\varepsilon}\right\}=\varepsilon .
$$

The main contribution of this work is the following. We introduce modifications of the statistics $\Delta_{T}$, so that their limit distributions do not depend on $\vartheta$ in the first problem and do not depend on $S_{0}(\cdot)$ in the second problem. Therefore the corresponding tests are asymptotically parameter free in the first case and asymptotically distribution free in the second case. These modifications essentially simplify the solution of the equations (1) and allow to choose the thresholds $c_{\varepsilon}$ before actually conducting the experiments. 
Let us briefly recall what happens in the analogous problems in the case of independent identically distributed observations $X_{1}, \ldots, X_{n}$. In the problem of the first type we have the following results. Suppose that under the basic hypothesis

$$
\mathcal{H}_{0} \quad: \quad X_{j} \sim F_{0}(\vartheta, x), \quad \vartheta \in \Theta,
$$

where $F_{0}(\cdot, x)$ is some known distribution function. The limit distribution of the Cramér-von Mises staistics (under hypothesis $\mathcal{H}_{0}$ )

$$
\Delta_{n}\left(X^{n}\right)=n \int_{-\infty}^{\infty}\left[\hat{F}_{n}(x)-F_{0}\left(\hat{\vartheta}_{n}, x\right)\right]^{2} \mathrm{~d} F_{0}\left(\hat{\vartheta}_{n}, x\right) \Longrightarrow \Delta(\vartheta)
$$

depends on $\vartheta$. Here $\hat{F}_{n}(x)$ is the empirical distribution function and $\hat{\vartheta}_{n}$ is some estimator. The choice of the threshold $c_{\varepsilon}$ for the GoF test

$$
\hat{\psi}_{n}=\mathbb{1}_{\left\{\Delta_{n}\left(X^{n}\right)>c_{\varepsilon}\right\}}
$$

can be a difficult problem, since $c_{\varepsilon}=c_{\varepsilon}(\vartheta)$ is solution of the equation

$$
\mathbf{P}_{\vartheta}\left\{\Delta(\vartheta)>c_{\varepsilon}\right\}=\varepsilon .
$$

It is well-known that for some distributions, say with shift and scale parameters like $F\left(\frac{x-\alpha}{\beta}\right)$, this limit can be asymptotically parameter free $(\mathrm{APF})$. For example, if the hypothesis is

$$
\mathcal{H}_{0} \quad: \quad X_{j} \sim \mathcal{N}\left(\alpha, \beta^{2}\right), \quad \vartheta=(\alpha, \beta) \in \Theta,
$$

then the limit distribution of the Cramér-von Mises staistics

$$
\Delta_{n}\left(X^{n}\right)=n \int_{-\infty}^{\infty}\left[\hat{F}_{n}(x)-F_{0}\left(\frac{x-\hat{\alpha}_{n}}{\hat{\beta}_{n}}\right)\right]^{2} \mathrm{~d} F_{0}\left(\frac{x-\hat{\alpha}_{n}}{\hat{\beta}_{n}}\right)
$$

does not depend on $\vartheta$ (see, e.g., [10], [5], [13]).

Here $F_{0}(x)$ is the distribution function of $N(0,1)$ random variable. Therefore the threshold $c_{\varepsilon}$ does not depend on $\vartheta$ and the test can be easily constructed. The similar statement for Pareto distribution was studied by Choulakian and Stephens [1] and another class of distributions was treated by Martynov [14].

The general case of ergodic diffusion processes with one-dimensional shift parameter was studied by Negri and Zhou [16]. They showed that the limit distribution of the Cramér-von Mises statistic does not depend on the unknown parameter. 


\section{Preliminaries}

We need some properties of the estimators $\hat{F}_{T}(x)$ and $\hat{f}_{T}(x)$, which we recall below. We assume that the trend $S(x)$ and the diffusion $\sigma(x)^{2}$ coefficients of the observed diffusion process

$$
\mathrm{d} X_{t}=S\left(X_{t}\right) \mathrm{d} t+\sigma\left(X_{t}\right) \mathrm{d} W_{t}, \quad X_{0}, \quad 0 \leq t \leq T
$$

satisfy the following conditions.

$\mathcal{E S}$. The function $S(\cdot)$ is locally bounded, the function $\sigma(\cdot)^{2}>0$ is continuous and for some $C>0$ the condition

$$
x S(x)+\sigma(x)^{2} \leq C\left(1+x^{2}\right)
$$

holds.

Under this condition the stochastic differential equation has a unique weak solution (see, e.g., [6]).

Let us denote

$$
V(x)=\int_{0}^{x} \exp \left\{-2 \int_{0}^{y} \frac{S(z)}{\sigma(z)^{2}} \mathrm{~d} z\right\} \mathrm{d} y
$$

and

$$
G(S)=\int_{-\infty}^{\infty} \sigma(y)^{-2} \exp \left\{2 \int_{0}^{x} \frac{S(y)}{\sigma(y)^{2}} \mathrm{~d} y\right\} \mathrm{d} x .
$$

The next condition is:

$\mathcal{R P}$. The functions $S(\cdot)$ and $\sigma(\cdot)^{2}$ are such that

$$
G(S)<\infty, \quad V(x) \longrightarrow \pm \infty \quad \text { as } \quad x \longrightarrow \pm \infty .
$$

Under this condition the diffusion process is ergodic, i.e., positive recurrent with the invariant density

$$
f(x)=\frac{1}{G(S) \sigma(x)^{2}} \exp \left\{2 \int_{0}^{x} \frac{S(y)}{\sigma(y)^{2}} \mathrm{~d} y\right\} .
$$

The empirical distribution function $\hat{F}_{T}(x)$ and the local time density estimator $\hat{f}_{T}(x)$ of the invariant density $f(x)$ are

$$
\hat{F}_{T}(x)=\frac{1}{T} \int_{0}^{T} \mathbb{1}_{\left\{X_{t}<x\right\}} \mathrm{d} t, \quad \hat{f}_{T}(x)=\frac{\Lambda_{T}(x)}{T \sigma(x)^{2}}
$$

where the local time $\Lambda_{T}(x)$ satisfies the equation (Tanaka-Meyer formula)

$$
\Lambda_{T}(x)=\left|X_{t}-x\right|-\left|X_{0}-x\right|-\int_{0}^{T} \operatorname{sgn}\left(X_{t}-x\right) \mathrm{d} X_{t} .
$$


Recall that these estimators are consistent, asymptotically normal and asymptotically efficient under the basic hypothesis (see [11]). The proof of these properties is based on the representations

$$
\begin{aligned}
\sqrt{T}\left(\hat{F}_{T}(x)-F(x)\right)= & \frac{2}{\sqrt{T}} \int_{0}^{T} \frac{F(x) F\left(X_{t}\right)-F\left(x \wedge X_{t}\right)}{\sigma\left(X_{t}\right) f\left(X_{t}\right)} \mathrm{d} W_{t} \\
& +\frac{2}{\sqrt{T}} \int_{X_{0}}^{X_{T}} \frac{F(y) F(x)-F(y \wedge x)}{\sigma(y)^{2} f(y)} \mathrm{d} y
\end{aligned}
$$

and

$$
\begin{aligned}
\sqrt{T}\left(\hat{f}_{T}(x)-f(x)\right)= & \frac{2 f(x)}{\sqrt{T}} \int_{0}^{T} \frac{F\left(X_{t}\right)-\mathbb{1}_{\left\{X_{t}>x\right\}}}{\sigma\left(X_{t} y\right) f\left(X_{t}\right)} \mathrm{d} W_{t} \\
& +\frac{2 f(x)}{\sqrt{T}} \int_{X_{0}}^{X_{T}} \frac{\mathbb{1}_{\{y>x\}}-F(y)}{\sigma(y)^{2} f(y)} \mathrm{d} y .
\end{aligned}
$$

Using these representations and the central limit theorem for stochastic integrals we obtain the limits in distribution

$$
\begin{aligned}
& \sqrt{T}\left(\hat{F}_{T}(x)-F(x)\right) \Longrightarrow 2 \int_{-\infty}^{\infty} \frac{F(y) F(x)-F(y \wedge x)}{\sigma(y) \sqrt{f(y)}} \mathrm{d} W(y), \\
& \sqrt{T}\left(\hat{f}_{T}(x)-f(x)\right) \Longrightarrow 2 f(x) \int_{-\infty}^{\infty} \frac{F(y)-\mathbb{1}_{\{y>x\}}}{\sigma(y) \sqrt{f(y)}} \mathrm{d} W(y),
\end{aligned}
$$

where $W(\cdot)$ is a two-sided Wiener process.

The estimator $\hat{f}_{T}(x)$ is the a.s. derivative of $\hat{F}_{T}(x)$. Indeed, using the equality (see [17])

$$
\int_{0}^{T} h\left(X_{t}\right) \mathrm{d} t=\int_{-\infty}^{\infty} h(y) \frac{\Lambda_{T}(y)}{\sigma(y)^{2}} \mathrm{~d} y
$$

we can write

$$
\hat{F}_{T}(x)=\int_{-\infty}^{\infty} \mathbb{I}_{\{y<x\}} \frac{\Lambda_{T}(y)}{T \sigma(y)^{2}} \mathrm{~d} y=\int_{-\infty}^{x} \hat{f}_{T}(y) \mathrm{d} y .
$$

As the local time is continuous with probability one we have the limit

$$
\lim _{\alpha \rightarrow 0} \frac{\hat{F}_{T}(x+\alpha)-\hat{F}_{T}(x)}{\alpha}=\lim _{\alpha \rightarrow 0} \frac{1}{\alpha} \int_{x}^{x+\alpha} \hat{f}_{T}(y) \mathrm{d} y=\hat{f}_{T}(x) .
$$

Therefore we can call the local time estimator the empirical density. It is easy to see that the representation (3) can be obtained from (2) through differentiating. 
Introduce the class $\mathcal{P}$ of locally bounded functions with polynomial majorants $(p>0)$

$$
\mathcal{P}=\left\{h(\cdot): \quad|h(y)| \leq C\left(1+|y|^{p}\right)\right\} .
$$

and the following condition:

$\mathcal{A}_{0}$. The functions $S(\cdot), \sigma(\cdot)^{ \pm 1} \in \mathcal{P}$ and

$$
\varlimsup_{|y| \rightarrow \infty} \operatorname{sgn}(y) \frac{S(y)}{\sigma(y)^{2}}<0 .
$$

Note that if $S(\cdot)$ and $\sigma(\cdot)$ satisfy $\mathcal{A}_{0}$ then the condition $\mathcal{R} \mathcal{P}$ is fulfilled.

Moreover, under condition $\mathcal{A}_{0}$ for any $p>0$ there exist $\kappa>0$ and $C>0$ such that

$$
\mathbf{E}\left|\sqrt{T}\left(\hat{f}_{T}(x)-f(x)\right)\right|^{p} \leq C e^{-\kappa|x|} .
$$

For the proof see Proposition 1.11, [11].

\section{Asymptotically Parameter Free Tests}

The first problem is the following. We observe an ergodic diffusion process $X^{T}=\left(X_{t}, 0 \leq t \leq T\right)$, which solves the equation

$$
\mathrm{d} X_{t}=S\left(X_{t}\right) \mathrm{d} t+\sigma \mathrm{d} W_{t}, \quad X_{0}, \quad 0 \leq t \leq T
$$

and we have to test the composite basic hypothesis:

$\mathcal{H}_{0}$ this process admits the stochastic differential

$$
\mathrm{d} X_{t}=-\beta \operatorname{sgn}\left(X_{t}-\alpha\right)\left|X_{t}-\alpha\right|^{\gamma} \mathrm{d} t+\sigma \mathrm{d} W_{t}, \quad X_{0}, \quad 0 \leq t \leq T,
$$

where $\vartheta=(\alpha, \beta)$ is the unknown parameter, $\vartheta \in \Theta=\left(a_{1}, a_{2}\right) \times\left(b_{1}, b_{2}\right), b_{1}>$ 0 .

against the nonparametric alternative:

$\mathcal{H}_{1}$ the observed process does not belong to this parametric family.

The parameters $\gamma \geq 0$ and $\sigma>0$ are assumed to be known.

Note that if $\gamma=1$, then we obtain Ornstein-Uhlenbeck process

$$
\mathrm{d} X_{t}=-\beta\left(X_{t}-\alpha\right) \mathrm{d} t+\sigma \mathrm{d} W_{t}, \quad X_{0}, \quad 0 \leq t \leq T
$$

and if $\gamma=0$ then the solution of (5) is the simple switching process

$$
\mathrm{d} X_{t}=-\beta \operatorname{sgn}\left(X_{t}-\alpha\right) \mathrm{d} t+\sigma \mathrm{d} W_{t}, \quad X_{0}, \quad 0 \leq t \leq T
$$


studied in [11], Section 3.4. For $\gamma=3$ we have the cubic trend

$$
\mathrm{d} X_{t}=-\beta\left(X_{t}-\alpha\right)^{3} \mathrm{~d} t+\sigma \mathrm{d} W_{t}, \quad X_{0}, \quad 0 \leq t \leq T .
$$

It is easy to verify that for $\beta>0, \gamma \geq 0$ this process is positive recurrent with the invariant density

$$
f(\vartheta, x)=\frac{\beta^{\frac{1}{\gamma+1}}}{G_{\gamma} \sigma^{\frac{2}{\gamma+1}}} \exp \left\{-\frac{2 \beta|x-\alpha|^{\gamma+1}}{(\gamma+1) \sigma^{2}}\right\} .
$$

The normalizing constant is

$$
G_{\gamma}=\left(\frac{2}{\gamma+1}\right)^{\frac{\gamma}{\gamma+1}} \Gamma\left(\frac{1}{\gamma+1}\right),
$$

where $\Gamma(\cdot)$ is the Gamma function. Below we denote by $f_{0}(x)=f\left(\vartheta_{0}, x\right)$ and $F_{0}(x)=F\left(\vartheta_{0}, x\right)$ the density and the distribution function corresponding to the values $\vartheta_{0}=(0,1), \sigma=1$ and we denote by $\xi$ the random variable with such distribution function.

It will be convenient to study the cases $\gamma \geq 1$ (including O-U process) and $0 \leq \gamma<\frac{1}{2}$ separately because the rates of convergence of the MLE $\hat{\alpha}_{T}$ in these two cases are essentially different. We defer the discussion of the complementary case $\gamma \in\left[\frac{1}{2}, 1\right)$ to section 3.3 below.

\subsection{Case $\gamma \geq 1$.}

Let us consider the following ergodic diffusion process as the basic model (under hypothesis $\mathcal{H}_{0}$ )

$$
\mathrm{d} X_{t}=-\beta \operatorname{sgn}\left(X_{t}-\alpha\right)\left|X_{t}-\alpha\right|^{\gamma} \mathrm{d} t+\sigma \mathrm{d} W_{t}, \quad X_{0}, \quad 0 \leq t \leq T,
$$

where $\vartheta=(\alpha, \beta) \in \Theta=\left(a_{1}, a_{2}\right) \times\left(b_{1}, b_{2}\right), b_{1}>0$ and $\gamma \geq 1$.

Recall that the MLE $\hat{\vartheta}_{T}=\left(\hat{\alpha}_{T}, \hat{\beta}_{T}\right)$ of the parameter $\vartheta$ is consistent and asymptotically normal. Moreover, the moments of this estimator converge too (see Theorem 2.8, [1] ):

$$
\sup _{\theta \in \Theta} T^{\frac{p}{2}} \mathbf{E}_{\vartheta}\left|\hat{\vartheta}_{T}-\vartheta\right|^{p} \leq C
$$

for any $p>0$. 


\subsubsection{The Test Based on Empirical Distribution Function}

We study the test

$$
\hat{\psi}_{T}\left(X^{T}\right)=\mathbb{1}_{\left\{\Delta_{T}\left(X^{T}\right)>c_{\varepsilon}\right\}},
$$

where the test statistic is

$$
\Delta_{T}\left(X^{T}\right)=\hat{\beta}_{T}^{\frac{2}{\gamma+1}} \sigma^{\frac{2(\gamma-1)}{\gamma+1}} T \int_{-\infty}^{\infty}\left[\hat{F}_{T}(x)-F\left(\hat{\vartheta}_{T}, x\right)\right]^{2} \mathrm{~d} F\left(\hat{\vartheta}_{T}, x\right) .
$$

Let us introduce the random variable

$$
\Delta=\int_{-\infty}^{\infty}\left[\Phi(y)+\frac{\Pi}{\gamma a} f_{0}(y)+\frac{y \Psi}{(\gamma+1) b} f_{0}(y)\right]^{2} f_{0}(y) \mathrm{d} y
$$

where

$$
\begin{aligned}
\Phi(y) & =2 \int_{-\infty}^{\infty} \frac{F_{0}(z) F_{0}(y)-F_{0}(z \wedge y)}{\sqrt{f_{0}(y)}} \mathrm{d} W(z), \\
\Pi & =\int_{-\infty}^{\infty}|z|^{\gamma-1} \sqrt{f_{0}(z)} \mathrm{d} W(z), \quad a=\mathbf{E}_{0}|\xi|^{2 \gamma-2}, \\
\Psi & =\int_{-\infty}^{\infty} \operatorname{sgn}(z)|z|^{\gamma} \sqrt{f_{0}(z)} \mathrm{d} W(z), \quad b=\mathbf{E}_{0}|\xi|^{2 \gamma} .
\end{aligned}
$$

Here $W(\cdot)$ is two-sided Wiener process. The constant $c_{\varepsilon}$ is defined by the equation

$$
\mathbf{P}\left(\Delta>c_{\varepsilon}\right)=\varepsilon .
$$

The distribution of the random variable $\Delta$ is not known in a closed form but the value $c_{\varepsilon}$ can be easily obtained with the help of the Monte Carlo simulations. Let us stress that this value is the same for all $\vartheta$ and therefore can be calculated before the experiment.

Our first result is

Theorem 1 The test $\hat{\psi}_{T}\left(X^{T}\right) \in \mathcal{K}_{\varepsilon}$.

Proof. We have the relation

$$
\begin{aligned}
\sqrt{T} & \left(\hat{F}_{T}(x)-F\left(\hat{\vartheta}_{T}, x\right)\right) \\
& =\sqrt{T}\left(\hat{F}_{T}(x)-F(\vartheta, x)\right)+\sqrt{T}\left(F(\vartheta, x)-F\left(\hat{\vartheta}_{T}, x\right)\right) \\
& =\eta_{T}(x)-\left(\sqrt{T}\left(\hat{\vartheta}_{T}-\vartheta\right), \frac{\partial F(\vartheta, x)}{\partial \vartheta}\right)+r_{T} .
\end{aligned}
$$


Here $\left(\sqrt{T}\left(\hat{\vartheta}_{T}-\vartheta\right), \frac{\partial F(\vartheta, x)}{\partial \vartheta}\right)$ is the usual scalar product in $R^{2}$ and stochastic process $\eta_{T}(x)$ is given by

$$
\eta_{T}(x)=\frac{2}{\sqrt{T}} \int_{0}^{T} \frac{F\left(\vartheta, X_{t}\right) F(\vartheta, x)-F\left(\vartheta, X_{t} \wedge x\right)}{\sigma f\left(\vartheta, X_{t}\right)} \mathrm{d} W_{t} .
$$

The convergence $r_{T} \rightarrow 0$ follows from the representation (2), the estimate

$$
\sup _{\vartheta \in \Theta} \mathbf{E}_{\vartheta}\left(\int_{0}^{\xi} \frac{F(\vartheta, y) F(\vartheta, x)-F(\vartheta, y \wedge x)}{f(\vartheta, y)} \mathrm{d} y\right)^{2}<C,
$$

which can be obtained by direct calculation (see [11], Theorem 4.6) and the estimate (77). Note that the density $f(\vartheta, x)$ has exponentially decreasing tails and all necessary estimates can be derived in the straightforward way.

Define the random functions

$$
\begin{aligned}
\hat{\eta}_{T}(x) & =\hat{\beta}_{T}^{\frac{1}{\gamma+1}} \sigma^{\frac{\gamma-1}{\gamma+1}}\left[\eta_{T}(x)-\left(\sqrt{T}\left(\hat{\vartheta}_{T}-\vartheta\right), \frac{\partial F(\vartheta, x)}{\partial \vartheta}\right)\right], \quad x \in R, \\
\eta_{0}(x) & =\Phi(y)+\frac{\Pi}{\gamma a} f_{0}(y)+\frac{y \Psi}{(\gamma+1) b} f_{0}(y), \quad x \in R .
\end{aligned}
$$

We have to verify that

$$
\int_{-\infty}^{\infty} \hat{\eta}_{T}(x)^{2} f\left(\hat{\vartheta}_{T}, x\right) \mathrm{d} x \Longrightarrow \int_{-\infty}^{\infty} \eta_{0}(x)^{2} f_{0}(x) \mathrm{d} x
$$

We start with the convergence of the finite dimensional distributions. The form of the invariant density (6) suggests the following change of variables

$$
Z_{t}=\frac{\beta^{\frac{1}{\gamma+1}}}{\sigma^{\frac{2}{\gamma+1}}}\left(X_{t}-\alpha\right) .
$$

This process satisfies the following stochastic differential

$$
\mathrm{d} Z_{t}=-\operatorname{sgn}\left(Z_{t}\right)\left|Z_{t}\right|^{\gamma} \mathrm{d}\left(t \beta^{\frac{2}{\gamma+1}} \sigma^{\frac{2(\gamma-1)}{\gamma+1}}\right)+\beta^{\frac{1}{\gamma+1}} \sigma^{\frac{\gamma-1}{\gamma+1}} \mathrm{~d} W_{t}, \quad 0 \leq t \leq T .
$$

Therefore, if we denote

$$
Y_{s}=Z_{s \beta^{-\frac{2}{\gamma+1}} \sigma^{-\frac{2(\gamma-1)}{\gamma+1}}}, \quad 0 \leq s=t \beta^{\frac{2}{\gamma+1}} \sigma^{\frac{2(\gamma-1)}{\gamma+1}} \leq T_{*}=T \beta^{\frac{2}{\gamma+1}} \sigma^{\frac{2(\gamma-1)}{\gamma+1}},
$$

the process $Y_{s}$ satisfies the equation

$$
\mathrm{d} Y_{s}=-\operatorname{sgn}\left(Y_{s}\right)\left|Y_{s}\right|^{\gamma} \mathrm{d} s+\mathrm{d} w_{s}, \quad Y_{0}, \quad 0 \leq s \leq T_{*},
$$


where $w_{s}=\beta^{\frac{1}{\gamma+1}} \sigma^{\frac{\gamma-1}{\gamma+1}} W_{t}$ (here $t=s \beta^{-\frac{2}{\gamma+1}} \sigma^{-\frac{2(\gamma-1)}{\gamma+1}}$ ) is another Wiener process. Obviously the process $Y_{s}$ is ergodic with the invariant density $f_{0}(x)$.

Let us define $y=\beta^{\frac{1}{\gamma+1}} \sigma^{-\frac{2}{\gamma+1}}(x-\alpha)$. Then we can write

$$
F(\vartheta, x)=F_{0}(y), \quad f(\vartheta, x)=\beta^{\frac{1}{\gamma+1}} \sigma^{-\frac{2}{\gamma+1}} f_{0}(y) .
$$

For the stochastic process $\eta_{T}(x)$ this change of variables gives the representation

$$
\begin{aligned}
\eta_{T}(x) & =\frac{2}{\sqrt{T}} \int_{0}^{T} \frac{F\left(\vartheta, X_{t}\right) F(\vartheta, x)-F\left(\vartheta, x \wedge X_{t}\right)}{\sigma f\left(\vartheta, X_{t}\right)} \mathrm{d} W_{t} \\
& =\frac{2}{\sqrt{T}} \int_{0}^{T} \frac{F_{0}\left(Z_{t}\right) F_{0}(y)-F_{0}\left(y \wedge Z_{t}\right)}{\beta^{\frac{1}{\gamma+1}} \sigma^{\frac{\gamma-1}{\gamma+1}} f_{0}\left(Z_{t}\right)} \mathrm{d} W_{t} \\
& =\frac{2 \beta^{-\frac{1}{\gamma+1}} \sigma^{-\frac{\gamma-1}{\gamma+1}}}{\sqrt{T_{*}}} \int_{0}^{T_{*}} \frac{F_{0}\left(Y_{s}\right) F_{0}(y)-F_{0}\left(y \wedge Y_{s}\right)}{f_{0}\left(Y_{s}\right)} \mathrm{d} w_{s} \\
& =\beta^{-\frac{1}{\gamma+1}} \sigma^{-\frac{\gamma-1}{\gamma+1}} \Phi_{T_{*}}(y),
\end{aligned}
$$

where the last equality defines the random function $\Phi_{T_{*}}(y)$.

Introduce the following integrals

$$
\begin{aligned}
& \pi_{T_{*}}=\frac{1}{\sqrt{T_{*}}} \int_{0}^{T_{*}}\left|Y_{s}\right|^{\gamma-1} \mathrm{~d} w_{s}, \quad \psi_{T_{*}}=\frac{1}{\sqrt{T_{*}}} \int_{0}^{T_{*}} \operatorname{sgn}\left(Y_{s}\right)\left|Y_{s}\right|^{\gamma} \mathrm{d} w_{s}, \\
& a_{T_{*}}=\frac{1}{T_{*}} \int_{0}^{T_{*}}\left|Y_{s}\right|^{2 \gamma-2} \mathrm{~d} s, \quad b_{T_{*}}=\frac{1}{T_{*}} \int_{0}^{T_{*}}\left|Y_{s}\right|^{2 \gamma} \mathrm{d} s, \\
& c_{T_{*}}=\frac{1}{T_{*}} \int_{0}^{T_{*}} \operatorname{sgn}\left(Y_{s}\right)\left|Y_{s}\right|^{2 \gamma-1} \mathrm{~d} s .
\end{aligned}
$$

Note that by the law of large numbers we have

$$
a_{T_{*}} \longrightarrow a, \quad b_{T_{*}} \longrightarrow b
$$

The invariant density $f_{0}(y)$ is a symmetric function and therefore

$$
c_{T_{*}} \longrightarrow \mathbf{E}_{\vartheta_{0}}\left(\operatorname{sgn}(\xi)|\xi|^{2 \gamma-1}\right)=0 .
$$

The random variables $\pi_{T_{*}}$ and $\psi_{T_{*}}$ are asymptotically normal by the central limit theorem

$$
\pi_{T_{*}} \Longrightarrow \Pi, \quad \psi_{T_{*}} \Longrightarrow \Psi
$$

and due to (10) they are asymptotically independent. 
The MLE $\hat{\vartheta}_{T}$ admits the following representation

$$
\sqrt{T}\left(\hat{\vartheta}_{T}-\vartheta\right)=\mathrm{I}_{T}(\vartheta)^{-1} \frac{1}{\sigma \sqrt{T}} \int_{0}^{T} \frac{\partial S\left(\vartheta, X_{t}\right)}{\partial \vartheta} \mathrm{d} W_{t}+o(1),
$$

where $\mathrm{I}_{T}(\vartheta)$ is the $2 \times 2$ matrix

$$
\mathrm{I}_{T}(\vartheta)=\frac{1}{T \sigma^{2}} \int_{0}^{T} \frac{\partial S\left(\vartheta, X_{t}\right)}{\partial \vartheta}\left(\frac{\partial S\left(\vartheta, X_{t}\right)}{\partial \vartheta}\right)^{\tau} \mathrm{d} t .
$$

Here $\tau$ means transposition. For the proof of this representation see Theorem 2.8 in [11] and Theorem 8.1 in [9]. The convergence (10) allows us to consider the information matrix as asymptotically diagonal.

Note that for the trend coefficient $S(\vartheta, x)=-\beta \operatorname{sgn}(x-\alpha)|x-\alpha|^{\gamma}$ we have the equality $\frac{\partial S(\vartheta, x)}{\partial \alpha}=\beta \gamma|x-\alpha|^{\gamma-1}$. We have

$$
\begin{aligned}
& \frac{1}{T \sigma^{2}} \int_{0}^{T}\left(\frac{\partial S\left(\vartheta, X_{t}\right)}{\partial \alpha}\right)^{2} \mathrm{~d} t=\gamma^{2} \sigma^{\frac{2(\gamma-3)}{\gamma+1}} \beta^{\frac{4}{\gamma+1}} a_{T_{*}}, \\
& \frac{1}{T \sigma^{2}} \int_{0}^{T}\left(\frac{\partial S\left(\vartheta, X_{t}\right)}{\partial \beta}\right)^{2} \mathrm{~d} t=\sigma^{\frac{2(\gamma-1)}{\gamma+1}} \beta^{-\frac{2 \gamma}{\gamma+1}} b_{T_{*}}
\end{aligned}
$$

and

$$
\begin{aligned}
& \frac{1}{\sqrt{T} \sigma} \int_{0}^{T} \frac{\partial S\left(\vartheta, X_{t}\right)}{\partial \alpha} \mathrm{d} W_{t}=\gamma \sigma^{\frac{\gamma-3}{\gamma+1}} \beta^{\frac{2}{\gamma+1}} \pi_{T_{*}}, \\
& \frac{1}{\sqrt{T} \sigma} \int_{0}^{T} \frac{\partial S\left(\vartheta, X_{t}\right)}{\partial \beta} \mathrm{d} W_{t}=-\sigma^{\frac{\gamma-1}{\gamma+1}} \beta^{-\frac{\gamma}{\gamma+1}} \psi_{T_{*}}
\end{aligned}
$$

Further

$$
\frac{\partial F(\vartheta, x)}{\partial \alpha}=-\frac{\beta^{\frac{1}{\gamma+1}}}{\sigma^{\frac{2}{\gamma+1}}} f_{0}(y), \quad \frac{\partial F(\vartheta, x)}{\partial \beta}=\frac{y}{\beta(\gamma+1)} f_{0}(y) .
$$

Therefore we can write

$$
\begin{aligned}
& \left(\sqrt{T}\left(\hat{\vartheta}_{T}-\vartheta\right), \frac{\partial F(\vartheta, x)}{\partial \vartheta}\right) \\
& =\sqrt{T}\left(\hat{\alpha}_{T}-\alpha\right) \frac{\partial F(\vartheta, x)}{\partial \alpha}+\sqrt{T}\left(\hat{\beta}_{T}-\beta\right) \frac{\partial F(\vartheta, x)}{\partial \beta} \\
& =-\beta^{-\frac{1}{\gamma+1}} \sigma^{\frac{1-\gamma}{\gamma+1}}\left[\frac{\pi_{T_{*}}}{\gamma a_{T_{*}}}+\frac{y \psi_{T_{*}}}{(\gamma+1) b_{T_{*}}}\right] f_{0}(y)+o(1) .
\end{aligned}
$$


This, in turn, allows us to write

$$
\begin{aligned}
\sqrt{T} & \left(\hat{F}_{T}(x)-F\left(\hat{\vartheta}_{T}, x\right)\right) \\
& =\beta^{-\frac{1}{\gamma+1}} \sigma^{\frac{1-\gamma}{\gamma+1}}\left[\Phi_{T_{*}}(y)+\frac{\pi_{T_{*}}}{\gamma a_{T_{*}}} f_{0}(y)+\frac{y \psi_{T_{*}}}{(\gamma+1) b_{T_{*}}} f_{0}(y)\right]+o(1) .
\end{aligned}
$$

Finally we obtain

$$
\begin{aligned}
\Delta_{T} & \left(X^{T}\right) \\
= & \int_{-\infty}^{\infty}\left[\Phi_{T_{*}}(y)+\frac{\pi_{T_{*}}}{\gamma a_{T_{*}}} f_{0}(y)+\frac{y \psi_{T_{*}}}{(\gamma+1) b_{T_{*}}} f_{0}(y)\right]^{2} f_{0}(y) \mathrm{d} y+o(1) .
\end{aligned}
$$

Now we can replace $a_{T_{*}}$ and $b_{T_{*}}$ with their limits $a$ and $b$ and denote

$$
\tilde{\eta}_{T_{*}}(y)=\Phi_{T_{*}}(y)+\frac{\pi_{T_{*}}}{\gamma a} f_{0}(y)+\frac{y \psi_{T_{*}}}{(\gamma+1) b} f_{0}(y) .
$$

We shall verify the convergence

$$
\int_{-\infty}^{\infty} \tilde{\eta}_{T_{*}}(y)^{2} f_{0}(y) \mathrm{d} y \Longrightarrow \int_{-\infty}^{\infty} \eta_{0}(y)^{2} f_{0}(y) \mathrm{d} y .
$$

To prove it, we shall check the following three conditions.

1. The finite dimensional distributions of $\tilde{\eta}_{T_{*}}(\cdot)$ converge, i.e., for any $k \geq 1$ and any $y_{1}, \ldots, y_{k}$ we have the convergence

$$
\left(\tilde{\eta}_{T_{*}}\left(y_{1}\right), \ldots, \tilde{\eta}_{T_{*}}\left(y_{k}\right)\right) \Longrightarrow\left(\eta_{0}\left(y_{1}\right), \ldots, \eta_{0}\left(y_{k}\right)\right) \text {. }
$$

2. There exists a constant $C_{1}>0$ such that

$$
\mathbf{E}_{\vartheta_{0}}\left|\tilde{\eta}_{T_{*}}\left(y_{2}\right)-\tilde{\eta}_{T_{*}}\left(y_{1}\right)\right|^{2} \leq C_{1}\left|y_{2}-y_{1}\right|
$$

3. There exist constants $C_{2}>0$ and $\kappa>0$ such

$$
\mathbf{E}_{\vartheta_{0}}\left|\tilde{\eta}_{T_{*}}(y)\right|^{2} \leq C_{2} e^{-\kappa|y|^{\gamma+1}}
$$

If these conditions hold, (12) follows from the results of [9]. Indeed, by Theorem A.22 in [9] integrals converge on any finite interval $[-L, L]$ and outside of this interval we can estimate the tail integrals as in the proof of the Theorem 1.5.6 [9]. 
By the central limit theorem for stochastic integral we obtain the desired joint asymptotic normality

$$
\left(\Phi_{T_{*}}\left(y_{1}\right), \ldots, \Phi_{T_{*}}\left(y_{k}\right), \pi_{T_{*}}, \psi_{T_{*}}\right) \Longrightarrow\left(\Phi\left(y_{1}\right), \ldots, \Phi\left(y_{k}\right), \Pi, \Psi\right),
$$

which proves convergence of the finite dimensional distributions of $\tilde{\eta}_{T}(\cdot)$. Further, for all $y_{1}, y_{2},\left|y_{2}-y_{1}\right| \leq 1$ we have the estimate $\left(y_{1}<y_{2}\right)$

$$
\begin{aligned}
\mathbf{E}_{\vartheta_{0}} \mid \tilde{\eta}_{T} & \left(y_{2}\right)-\left.\tilde{\eta}_{T}\left(y_{1}\right)\right|^{2} \leq 3 \mathbf{E}_{\vartheta_{0}}\left|\Phi_{T_{*}}\left(y_{2}\right)-\Phi_{T_{*}}\left(y_{1}\right)\right|^{2} \\
& +\frac{3}{(\gamma+1)^{2} b^{2}}\left|y_{2} f_{0}\left(y_{2}\right)-y_{1} f_{0}\left(y_{1}\right)\right|^{2} \mathbf{E}_{\vartheta_{0}} \psi_{T_{*}}^{2} \\
& +\frac{3}{\gamma^{2} a^{2}}\left|f_{0}\left(y_{2}\right)-f_{0}\left(y_{1}\right)\right|^{2} \mathbf{E}_{\vartheta_{0}} \pi_{T_{*}}^{2} \leq C\left|y_{2}-y_{1}\right|^{2}
\end{aligned}
$$

because

$$
\begin{aligned}
\mathbf{E}_{\vartheta_{0}} \mid \Phi_{T_{*}} & \left(y_{2}\right)-\left.\Phi_{T_{*}}\left(y_{1}\right)\right|^{2}=4 \int_{-\infty}^{y_{1}} \frac{F_{0}(z)^{2}\left[F_{0}\left(y_{2}\right)-F_{0}\left(y_{1}\right)\right]^{2}}{f_{0}(z)} \mathrm{d} z \\
& +4 \int_{y_{2}}^{\infty} \frac{F_{0}(z)^{2}\left[F_{0}\left(y_{2}\right)-F_{0}\left(y_{1}\right)\right]^{2}}{f_{0}(z)} \mathrm{d} z \\
& +4 \int_{y_{1}}^{y_{2}} \frac{\left(F_{0}(z)\left[F_{0}\left(y_{2}\right)-F_{0}\left(y_{1}\right)\right]-F_{0}(z)+F_{0}\left(y_{1}\right)\right)^{2}}{f_{0}(z)} \mathrm{d} z \\
\leq & C\left|y_{2}-y_{1}\right|^{2} .
\end{aligned}
$$

Therefore, we obtain (13). To prove (14), write

$$
\begin{aligned}
& \mathbf{E}_{\vartheta_{0}} \tilde{\eta}_{T}(y)^{2} \leq 3 \mathbf{E}_{\vartheta_{0}} \Phi_{T_{*}}(y)^{2}+\frac{3}{(\gamma+1)^{2} b^{2}} y^{2} f_{0}(y)^{2} \mathbf{E}_{\vartheta_{0}} \Psi_{T_{*}}^{2}+\frac{3}{\gamma^{2} a^{2}} f_{0}(y)^{2} \mathbf{E}_{\vartheta_{0}} \Pi_{T_{*}}^{2} \\
& \quad \leq 12 \int_{-\infty}^{\infty} \frac{\left[F_{0}(z) F_{0}(y)-F_{0}(z \wedge y)\right]^{2}}{f_{0}(z)} \mathrm{d} z+C\left(1+y^{2}\right) f_{0}(y)^{2} .
\end{aligned}
$$

Further, using the same arguments as in [11], Example 4.1.3, we obtain the estimate

$$
\left(F_{0}(y)-1\right)^{2} \int_{-\infty}^{y} \frac{F_{0}(z)^{2}}{f_{0}(z)} \mathrm{d} z+F_{0}(y)^{2} \int_{y}^{\infty} \frac{\left[F_{0}(z)-1\right]^{2}}{f_{0}(z)} \mathrm{d} z \leq C e^{-\kappa|y|^{\gamma+1}}
$$

with some constants $C>0, \kappa>0$. For example, for the large values of $z$ we can write

$$
\begin{gathered}
\frac{\left[F_{0}(z)-1\right]^{2}}{f_{0}(z)}=\left(\int_{z}^{\infty} \exp \left\{-c u^{\gamma+1}+\frac{c}{2} z^{\gamma+1}\right\} \mathrm{d} u\right)^{2} \\
\leq\left(\int_{z}^{\infty} \exp \left\{-\frac{c}{2} u^{\gamma+1}\right\} \mathrm{d} u\right)^{2} \leq C e^{-c z^{\gamma+1}}
\end{gathered}
$$


and so on.

Therefore the conditions of the weak convergence of integrals are verified and the test $\hat{\psi}_{T} \in \mathcal{K}_{\varepsilon}$.

The consistency of this test is implied by the elementary inequalities as follows. Suppose that the trend coefficient $S(x)$ of the observed process (4) does not belong to the given parametric family $S(\vartheta, x), \vartheta \in\left[a_{1}, a_{2}\right] \times\left[b_{1}, b_{2}\right]$. It is known (Proposition 2.36, [1] ) that the MLE $\hat{\vartheta}_{T}$ converges to the value $\hat{\vartheta}$ which minimizes the Kullback-Leibner distance between the parametric family and the true distribution:

$$
\hat{\vartheta}=\arg \inf _{\vartheta} \mathbf{E}_{S}\left(\frac{S\left(\xi_{*}\right)-S\left(\vartheta, \xi_{*}\right)}{\sigma\left(\xi_{*}\right)}\right)^{2}
$$

Here the random variable $\xi_{*}$ has the invariant density function $f_{S}(x)$. It can be shown that

$$
\int_{R}\left[\hat{F}_{T}(x)-F\left(\hat{\vartheta}_{T}, x\right)\right]^{2} \mathrm{~d} F\left(\hat{\vartheta}_{T}, x\right) \rightarrow \int_{R}[F(x)-F(\hat{\vartheta}, x)]^{2} \mathrm{~d} F(\hat{\vartheta}, x)>0 .
$$

Therefore for the statistic $\Delta_{T}$ we have

$$
\Delta_{T} \longrightarrow \infty \quad \text { and } \quad \mathbf{P}_{S}\left\{\Delta_{T}>c_{\varepsilon}\right\} \rightarrow 1
$$

Hence the test is consistent.

\subsubsection{The Test Based on Empirical Density}

Now we study the test

$$
\tilde{\psi}_{T}\left(X^{T}\right)=\mathbb{I}_{\left\{\delta_{T}\left(X^{T}\right)>c_{\varepsilon}\right\}},
$$

where the test statistic is

$$
\delta_{T}\left(X^{T}\right)=\sigma^{2} T \int_{-\infty}^{\infty}\left[\hat{f}_{T}(x)-f\left(\hat{\vartheta}_{T}, x\right)\right]^{2} \mathrm{~d} F\left(\hat{\vartheta}_{T}, x\right) .
$$

Let us denote $\zeta_{T}(\vartheta, x)=\sqrt{T}\left(\hat{f}_{T}(x)-f(\vartheta, x)\right)$ and write

$$
\begin{aligned}
\sqrt{T}\left(\hat{f}_{T}(x)-f\left(\hat{\vartheta}_{T}, x\right)\right)= & \zeta_{T}(\vartheta, x)-\sqrt{T}\left(\hat{\alpha}_{T}-\alpha\right) \frac{\partial f(\vartheta, x)}{\partial \alpha} \\
& -\sqrt{T}\left(\hat{\beta}_{T}-\beta\right) \frac{\partial f(\vartheta, x)}{\partial \beta}+o(1) .
\end{aligned}
$$


Using the same arguments as above we obtain the representations

$$
\begin{aligned}
\zeta_{T}(\vartheta, x) & =\frac{2 f_{0}(y)}{\sigma \sqrt{T_{*}}} \int_{0}^{T_{*}} \frac{F_{0}\left(Y_{s}\right)-\mathbb{I}_{\left\{Y_{s}>y\right\}}}{f_{0}\left(Y_{s}\right)} \mathrm{d} w_{s}+o(1) \\
& =\sigma^{-1} \tilde{\Phi}_{T_{*}}(y) f_{0}(y)+o(1), \\
\frac{\partial f(\vartheta, x)}{\partial \alpha} & =-2 \beta^{\frac{2}{\gamma+1}} \sigma^{-\frac{2}{\gamma+1}} \operatorname{sgn}(y)|y|^{\gamma} f_{0}(y), \\
\frac{\partial f(\vartheta, x)}{\partial \beta} & =-\frac{\beta^{-\frac{\gamma}{\gamma+1}} \sigma^{-\frac{2}{\gamma+1}}}{\gamma+1}\left[1-2|y|^{\gamma+1}\right] f_{0}(y) .
\end{aligned}
$$

These equalities together with the representations of the estimators $\hat{\alpha}_{T}$ and $\hat{\beta}_{T}$ allow us to write

$$
\begin{aligned}
\sigma \sqrt{T} & \left(\hat{f}_{T}(x)-f\left(\hat{\vartheta}_{T}, x\right)\right) \\
& =\left[\tilde{\Phi}_{T_{*}}(y)+2 \operatorname{sgn}(y)|y|^{\gamma} \frac{\pi_{T_{*}}}{\gamma a}+\left[1-2|y|^{\gamma+1}\right] \frac{\psi_{T_{*}}}{(\gamma+1) b}\right] f_{0}(y)+o(1) .
\end{aligned}
$$

Using the same arguments as in the section 3.1.1 the following convergence

$$
\delta_{T} \Longrightarrow \delta=\int_{-\infty}^{\infty} \zeta_{0}(y)^{2} f_{0}(y) \mathrm{d} y
$$

can be proved. Here

$$
\begin{aligned}
& \zeta_{0}(y)=\left[\tilde{\Phi}(y)+2 \operatorname{sgn}(y)|y|^{\gamma} \frac{\Pi}{\gamma a}+\left[1-2|y|^{\gamma+1}\right] \frac{\Psi}{(\gamma+1) b}\right] f_{0}(y), \\
& \tilde{\Phi}(y)=2 \int_{-\infty}^{\infty} \frac{F_{0}(z)-\mathbb{I}_{\{z>y\}}}{\sqrt{f_{0}(z)}} \mathrm{d} W(z) .
\end{aligned}
$$

Hence the test $\tilde{\psi}_{T}=\mathbb{I}_{\left\{\delta_{T}>c_{\varepsilon}\right\}}$ is APF. The threshold $c_{\varepsilon}$ is defined by the equation

$$
\mathbf{P}\left\{\delta>c_{\varepsilon}\right\}=\varepsilon
$$

and therefore it belongs to $\mathcal{K}_{\varepsilon}$.

\subsection{Case $0 \leq \gamma<\frac{1}{2}$.}

If we observe (under hypothesis $\mathcal{H}_{0}$ ) the same equation

$$
\mathrm{d} X_{t}=-\beta \operatorname{sgn}\left(X_{t}-\alpha\right)\left|X_{t}-\alpha\right|^{\gamma} \mathrm{d} t+\sigma \mathrm{d} W_{t}, \quad X_{0}, \quad 0 \leq t \leq T .
$$

but with $\gamma \in\left[0, \frac{1}{2}\right)$, then the main difference with $\gamma \geq 1$ is due to the rate of convergence of the MLE $\hat{\alpha}_{T}$. As the rate is faster than $\sqrt{T}$ the contribution 
of this estimator to the limit distribution of test statistic is negligeable. This property of the test statisics was mentioned by Darling [4].

Recall that in the case $\gamma=0$ (simple switching)

$$
\mathrm{d} X_{t}=-\beta \operatorname{sgn}\left(X_{t}-\alpha\right) \mathrm{d} t+\sigma \mathrm{d} W_{t}, \quad X_{0}, \quad 0 \leq t \leq T .
$$

we have the convergence

$$
T\left(\hat{\alpha}_{T}-\alpha\right) \Longrightarrow \hat{u}, \quad Z(\hat{u})=\sup _{u} Z(u)
$$

where

$$
Z(u)=\exp \left\{W\left(u \gamma_{\vartheta}\right)-\frac{\left|u \gamma_{\vartheta}\right|}{2}\right\} .
$$

Here $W(\cdot)$ is double sided Wiener process and $\gamma_{\vartheta}>0$ is some constant (see details in [11, Section 3.4). Moreover, we have the convergence of moments too: for any $p>0$

$$
\mathbf{E}_{\vartheta}\left|T\left(\hat{\alpha}_{T}-\alpha\right)\right|^{p} \longrightarrow \mathbf{E}_{\vartheta}|\hat{u}|^{p} .
$$

Therefore, if we repeat the proofs above, we shall see that

$$
\sqrt{T}\left(\hat{\alpha}_{T}-\alpha\right) \frac{\partial F(\vartheta, x)}{\partial \alpha}=O\left(\frac{1}{\sqrt{T}}\right)
$$

and

$$
\sqrt{T}\left(\hat{\alpha}_{T}-\alpha\right) \frac{\partial f(\vartheta, x)}{\partial \alpha}=O\left(\frac{1}{\sqrt{T}}\right) .
$$

Of course, we have to be careful with the second term because the invariant density is

$$
f(\vartheta, x)=\frac{\beta}{\sigma^{2}} \exp \left\{-\frac{2 \beta}{\sigma^{2}}|x-\alpha|\right\}
$$

and the derivative is not continuous. However, the function $f(\vartheta, x)$ is absolutely continuous and this is sufficient for the proof.

The Cramér-von Mises type statistics are

$$
\begin{aligned}
\Delta_{T}\left(X^{T}\right) & =\frac{\hat{\beta}_{T}^{2}}{\sigma^{2}} T \int_{-\infty}^{\infty}\left[\hat{F}_{T}(x)-F\left(\hat{\vartheta}_{T}, x\right)\right]^{2} \mathrm{~d} F\left(\hat{\vartheta}_{T}, x\right), \\
\delta_{T}\left(X^{T}\right) & =\sigma^{2} T \int_{-\infty}^{\infty}\left[\hat{f}_{T}(x)-f\left(\hat{\vartheta}_{T}, x\right)\right]^{2} \mathrm{~d} F\left(\hat{\vartheta}_{T}, x\right),
\end{aligned}
$$

and their limits are

$$
\begin{aligned}
\Delta_{T}\left(X^{T}\right) & \Longrightarrow \int_{-\infty}^{\infty}\left[\Phi(y)+y f_{0}(y) \frac{\Psi}{(\gamma+1) b}\right]^{2} f_{0}(y) \mathrm{d} y \\
\delta_{T}\left(X^{T}\right) & \Longrightarrow \int_{-\infty}^{\infty}\left[\tilde{\Phi}(y)+[1-2|y|] \frac{\Psi}{(\gamma+1) b}\right]^{2} f_{0}(y)^{3} \mathrm{~d} y .
\end{aligned}
$$


Hence the corresponding tests belong to $\mathcal{K}_{\varepsilon}$.

If $\gamma \in\left(0, \frac{1}{2}\right)$, then we have a different limit

$$
T^{\frac{1}{2 \gamma+1}}\left(\hat{\alpha}_{T}-\alpha\right) \Longrightarrow \hat{u}, \quad Z(\hat{u})=\sup _{u} Z(u)
$$

with $Z(u)=\exp \left\{W^{H}\left(u \Gamma_{\vartheta}\right)-\frac{\left|u \Gamma_{\vartheta}\right|^{2 H}}{2}\right\}$. Here $W^{H}(\cdot)$ is two sided fractional Brownian motion, $H=\kappa+\frac{1}{2}$ (Hurst parameter) and $\Gamma_{\vartheta}$ is some constant. We have the convergence of moments too: for any $p>0$

$$
T^{\frac{p}{2 \gamma+1}} \mathbf{E}_{\vartheta}\left|\left(\hat{\vartheta}_{T}-\vartheta\right)\right|^{p} \longrightarrow \mathbf{E}_{\vartheta}|\hat{u}|^{p}
$$

For the proofs see [2] or [11], Section 3.2.

Hence once again we can use the tests $\hat{\psi}_{T}$ and $\tilde{\psi}_{T}$ and the limits of the test statistics are obtained by setting $\Pi \equiv 0$ in $\Delta$ and $\delta$ respectively.

\subsection{Discussion}

The case $\gamma \in\left[\frac{1}{2}, 1\right)$ was not included in this study because the appropriate properties of the MLE $\hat{\alpha}_{T}$ are available only in the cases $\gamma \in\left[0, \frac{1}{2}\right)$ [2] and $\gamma \geq 1$ [11]. In the case $\gamma \in\left(\frac{1}{2}, 1\right)$ the derivative of the trend with respect to parameter $\alpha$ is no more locally bounded, but the singularity at the point $x=\alpha$ is integrable and the proof presented in [11, Theorem 2.8 can be carried out. Note that the Fisher information is bounded. Therefore in this case we obtain the same result as for $\gamma \geq 1$. This is not the case if $\gamma=\frac{1}{2}$ and for this model we need a special study. Note that the rate of convergence of the MLE is better than $\sqrt{T}$ and the limit distribution of the test statistic have to be the same as for $\gamma \in\left(0, \frac{1}{2}\right)$.

Note that our proofs (strengthened up to the weak convergence in $\mathcal{C}_{0}(R)$ ) imply the following limits for the Kolmogorov-Smirnov type statistics

$$
\begin{aligned}
& \tilde{\Delta}_{T}\left(X^{T}\right)=\hat{\beta}_{T}^{\frac{1}{\gamma+1}} \sigma^{\frac{(\gamma-1)}{\gamma+1}} \sup _{x} \sqrt{T}\left|\hat{F}_{T}(x)-F\left(\hat{\vartheta}_{T}, x\right)\right| \Longrightarrow \sup _{y}\left|\eta_{0}(y)\right|, \\
& \tilde{\delta}_{T}\left(X^{T}\right)=\sigma \sup _{x} \sqrt{T}\left|\hat{f}_{T}(x)-f\left(\hat{\vartheta}_{T}, x\right)\right| \Longrightarrow \sup _{y}\left|\zeta_{0}(y)\right|
\end{aligned}
$$

where the limit distributions do not depend on $\vartheta$. Hence the goodness of fit tests based on these statistics are APF. It will be interesting to consider other models with APF tests. 


\section{Asymptotically Distribution Free Tests}

Now we consider the second statement of the goodness of fit hypotheses testing problem. The basic hypothesis $\mathcal{H}_{0}$ is simple: the observed diffusion process satisfies the stochastic differential equation

$$
\mathrm{d} X_{t}=S_{0}\left(X_{t}\right) \mathrm{d} t+\sigma\left(X_{t}\right) \mathrm{d} W_{t}, \quad X_{0}, \quad 0 \leq t \leq T,
$$

where $S_{0}(\cdot)$ and $\sigma(\cdot)$ are known functions. We assume that the conditions $\mathcal{E S}$ and $\mathcal{A}_{0}$ hold. Therefore, the stochastic process are ergodic with the invariant density

$$
f_{S_{0}}(x)=\frac{1}{G\left(S_{0}\right) \sigma(x)^{2}} \exp \left\{2 \int_{0}^{x} \frac{S_{0}(y)}{\sigma(y)^{2}} \mathrm{~d} y\right\} .
$$

Of course, we can use the Cramér-von Mises type statistics based on the empirical density

$$
\delta_{T}\left(X^{T}\right)=T \int_{-\infty}^{\infty}\left[\hat{f}_{T}(x)-f_{S_{0}}(x)\right]^{2} \mathrm{~d} F_{S_{0}}(x),
$$

but its limit under hypothesis is

$$
\delta\left(S_{0}\right)=\int_{-\infty}^{\infty} \zeta\left(S_{0}, x\right)^{2} \mathrm{~d} F_{S_{0}}(x)
$$

where

$$
\zeta\left(S_{0}, x\right)=2 f_{S_{0}}(x) \int_{-\infty}^{\infty} \frac{F_{S_{0}}(y)-\mathbb{I}_{\{y>x\}}}{\sigma(y) \sqrt{f_{S_{0}}(y)}} \mathrm{d} W(y)
$$

is the limit in distribution of the normalized difference

$$
\zeta_{T}\left(S_{0}, x\right)=\sqrt{T}\left(\hat{f}_{T}(x)-f_{S_{0}}(x)\right)
$$

(see the representation (30) ). tion

Therefore, for the test $\phi_{T}\left(X^{T}\right)=\mathbb{I}_{\left\{\delta_{T}\left(X^{T}\right)>c_{\varepsilon}\right\}}$ we have to solve the equa-

$$
\mathbf{P}_{S_{0}}\left(\delta\left(S_{0}\right)>c_{\varepsilon}\right)=\varepsilon
$$

and we see that the threshold $c_{\varepsilon}=c_{\varepsilon}\left(S_{0}\right)$.

Recall that for the i.i.d. observations the limit of the corresponding statistics based on empirical distribution function is

$$
\Delta_{n}=n \int_{-\infty}^{\infty}\left[\hat{F}_{n}(x)-F_{0}(x)\right]^{2} \mathrm{~d} F_{0}(x) \Longrightarrow \Delta=\int_{0}^{1} B(t)^{2} \mathrm{~d} t,
$$


where $B(t), 0 \leq t \leq 1$ is the Brownian bridge. Hence the test $\hat{\phi}_{n}=\mathbb{1}_{\left\{\Delta_{n}>c_{\varepsilon}\right\}}$ with $c_{\varepsilon}$ from the equation $\mathbf{P}\left(\Delta>c_{\varepsilon}\right)=\varepsilon$ belongs to the class $\mathcal{K}_{\varepsilon}$. Tests based on statistics with limit distributions independent of the model under hypothesis are called asymptotically distribution free (ADF). There are several works devoted to the construction of ADF tests for ergodic diffusion processes observed in continuous time. We can mention here [7], [8, [11, [3], [15],[12], but the connection of these tests with the classical Cramér-von Mises and Kolmogorov-Smirnov is not evident. One exception is the work [12], where a linear transformation of the normalized deviations $\zeta_{T}(\cdot)$ and $\eta_{T}(\cdot)$ allowed construction of ADF tests. Unfortunately, the proof in [12] is not satisfactory, since it used a property of the time change in the Wiener integral, which is not always true. That is why we decided to suggest another linear transformation which leads to ADF test.

Therefore, our goal is to find a linear transformation $L\left(\zeta_{T}\right)$ of the random function $\zeta_{T}(x)$ such that

$$
\delta_{T}\left(X^{T}\right)=\int_{-\infty}^{\infty}\left[L\left(\zeta_{T}\right)(x)\right]^{2} \mathrm{~d} F_{S_{0}}(x) \Longrightarrow \int_{0}^{1} w(t)^{2} \mathrm{~d} t,
$$

where $w(t), 0 \leq t \leq 1$ is a Wiener process. Then obviously the test $\hat{\psi}_{T}\left(X^{T}\right)=\mathbb{1}_{\left\{\delta_{T}\left(X^{T}\right)>c_{\varepsilon}\right\}}$ with $c_{\varepsilon}$ from the equation

$$
\mathbf{P}\left\{\int_{0}^{1} w(t)^{2} \mathrm{~d} t>c_{\varepsilon}\right\}=\varepsilon
$$

will be ADF.

Let us rewrite the stochastic integral as follows

$$
\begin{aligned}
\int_{-\infty}^{\infty} & \frac{F_{S_{0}}(y)-\mathbb{I}_{\{y>x\}}}{\sigma(y) \sqrt{f_{S_{0}}(y)}} \mathrm{d} W(y) \\
& =\int_{-\infty}^{\infty} \frac{F_{S_{0}}(y)-\mathbb{I}_{\left\{F_{S_{0}}(y)>F_{S_{0}}(x)\right\}}}{\sigma(y) f_{S_{0}}(y)} \sqrt{f_{S_{0}}(y)} \mathrm{d} W(y) \\
& =\int_{-\infty}^{\infty} \frac{F_{S_{0}}(y)-\mathbb{I}_{\left\{F_{S_{0}}(y)>F_{S_{0}}(x)\right\}}}{\sigma(y) f_{S_{0}}(y)} \mathrm{d} w\left(F_{S_{0}}(y)\right) \\
& =\int_{0}^{1} \frac{s-\mathbb{I}_{\{s>t\}}}{a(s) b(s)} \mathrm{d} w(s) \\
& =\int_{0}^{t} \frac{s}{a(s) b(s)} \mathrm{d} w(s)+\int_{t}^{1} \frac{s-1}{a(s) b(s)} \mathrm{d} w(s) \equiv u(t),
\end{aligned}
$$

where $w(t), 0 \leq t \leq 1$ is a Wiener process

$$
w(t)=\int_{-\infty}^{F_{S_{0}}^{-1}(t)} \sqrt{f_{S_{0}}(y)} \mathrm{d} W(y)
$$


and we denoted

$$
F_{S_{0}}(y)=s, \quad F_{S_{0}}(x)=t, \quad a(s)=\sigma\left(F_{S_{0}}^{-1}(s)\right), \quad b(s)=f_{S_{0}}\left(F_{S_{0}}^{-1}(s)\right) .
$$

Here $F_{S_{0}}^{-1}(s)$ is the function inverse to $F_{S_{0}}(y)$, i.e., the solution $y$ of the equation $F_{S_{0}}(y)=s$. Note that under our assumptions the function $F_{S_{0}}(y)$ is strictly increasing.

We can write

$$
\mathrm{d} u(t)=\frac{t}{a(t) b(t)} \mathrm{d} w(t)-\frac{t-1}{a(t) b(t)} \mathrm{d} w(t)=\frac{1}{a(t) b(t)} \mathrm{d} w(t) .
$$

Hence the integral (understood in the mean square sense)

$$
\int_{0}^{t} a(s) b(s) \mathrm{d} u(s)=w(t)
$$

provides us the desired transformation. Indeed, we have

$$
u(t)=\frac{\zeta\left(S_{0}, F_{S_{0}}^{-1}(t)\right)}{2 f_{S_{0}}\left(F_{S_{0}}^{-1}(t)\right)}
$$

and

$$
\int_{0}^{t} a(s) b(s) \mathrm{d} u(s)=\int_{-\infty}^{x} \sigma(y) f_{S_{0}}(y) \mathrm{d}\left[\frac{\zeta\left(S_{0}, y\right)}{2 f_{S_{0}}(y)}\right]=w\left(F_{S_{0}}(x)\right)=w(t) .
$$

Therefore, we can write

$$
\delta=\int_{-\infty}^{\infty}\left(\int_{-\infty}^{x} \sigma(y) f_{S_{0}}(y) \mathrm{d}\left[\frac{\zeta\left(S_{0}, y\right)}{2 f_{S_{0}}(y)}\right]\right)^{2} \mathrm{~d} F_{S_{0}}(x)=\int_{0}^{1} w(t)^{2} \mathrm{~d} t .
$$

This equality suggests the statistic

$$
\hat{\delta}_{T}\left(X^{T}\right)=\int_{-\infty}^{\infty}\left(\int_{-\infty}^{x} \sigma(y) f_{S_{0}}(y) \mathrm{d}\left[\frac{\zeta_{T}\left(S_{0}, y\right)}{2 f_{S_{0}}(y)}\right]\right)^{2} \mathrm{~d} F_{S_{0}}(x),
$$

where we have to define the integral with respect to the normalized empirical density

$$
\int_{a}^{b} h(x) \mathrm{d}\left[\frac{\zeta_{T}(x)}{2 f_{S_{0}}(x)}\right]
$$

If we verify the convergence $\hat{\delta}_{T}\left(X^{T}\right) \Longrightarrow \delta$, then the corresponding test

$$
\hat{\psi}_{T}\left(X^{T}\right)=\mathbb{I}_{\left\{\hat{\delta}_{T}\left(X^{T}\right)>c_{\varepsilon}\right\}}, \quad \mathbf{P}\left\{\delta>c_{\varepsilon}\right\}=\varepsilon
$$


will be ADF.

Using the representation (3) for any piece-wise continuous function $h(x)$ with bounded support and partition $a=x_{0}<x_{1}<\ldots<x_{m}=b$ we can write

$$
\begin{aligned}
\sum_{x_{i}} h\left(\tilde{x}_{i}\right)\left[\frac{\zeta_{T}\left(x_{i+1}\right)}{2 f_{S_{0}}\left(x_{i+1}\right)}-\frac{\zeta_{T}\left(x_{i}\right)}{2 f_{S_{0}}\left(x_{i}\right)}\right] \\
=\frac{1}{\sqrt{T}} \int_{0}^{T} \frac{\sum_{x_{i}} h\left(\tilde{x}_{i}\right) \mathbb{1}_{\left\{x_{i}<X_{t} \leq x_{i+1}\right\}}}{\sigma\left(X_{t}\right) f_{S_{0}}\left(X_{t}\right)} \mathrm{d} W_{t} \\
-\frac{1}{\sqrt{T}} \int_{X_{0}}^{X_{T}} \frac{\sum_{x_{i}} h\left(\tilde{x}_{i}\right) \mathbb{I}_{\left\{x_{i}<y \leq x_{i+1}\right\}}}{\sigma(y) f_{S_{0}}(y)} \mathrm{d} y .
\end{aligned}
$$

Therefore as $\max \left|x_{i+1}-x_{i}\right| \rightarrow 0$ we obtain the limit

$$
\begin{aligned}
\lim _{m \rightarrow \infty} & \sum_{x_{i}} h\left(\tilde{x}_{i}\right)\left[\frac{\zeta_{T}\left(x_{i+1}\right)}{2 f_{S_{0}}\left(x_{i+1}\right)}-\frac{\zeta_{T}\left(x_{i}\right)}{2 f_{S_{0}}\left(x_{i}\right)}\right] \\
& =\frac{1}{\sqrt{T}} \int_{0}^{T} \frac{h\left(X_{t}\right) \mathbb{I}_{\left\{a<X_{t} \leq b\right\}}}{\sigma\left(X_{t}\right) f_{S_{0}}\left(X_{t}\right)} \mathrm{d} W_{t}-\frac{1}{\sqrt{T}} \int_{X_{0}}^{X_{T}} \frac{h(y) \mathbb{I}_{\{a<y \leq b\}}}{\sigma(y)^{2} f_{S_{0}}(y)} \mathrm{d} y .
\end{aligned}
$$

If $a=-\infty$ (as in our case) then this limit exists for a class of functions vanishing at $-\infty$ because $\sigma(y) f_{S_{0}}(y) \rightarrow 0$ as $|y| \rightarrow \infty$.

Recall that in our case $h(x)=\sigma(x) f_{S_{0}}(x)$ and the integral

$$
\begin{aligned}
\int_{-\infty}^{x} \sigma(y) f_{S_{0}}(y) \mathrm{d}\left[\frac{\zeta_{T}\left(S_{0}, y\right)}{2 f_{S_{0}}(y)}\right] \\
\quad=\frac{1}{\sqrt{T}} \int_{0}^{T} \mathbb{I}_{\left\{X_{t} \leq x\right\}} \mathrm{d} W_{t}-\frac{1}{\sqrt{T}} \int_{X_{0}}^{X_{T}} \frac{\mathbb{1}_{\{y \leq x\}}}{\sigma(y)} \mathrm{d} y .
\end{aligned}
$$

Therefore,

$$
\begin{aligned}
\hat{\delta}_{T}\left(X_{T}\right) & =\int_{-\infty}^{\infty}\left(\frac{1}{\sqrt{T}} \int_{0}^{T} \mathbb{I}_{\left\{X_{t} \leq x\right\}} \mathrm{d} W_{t}-\frac{H\left(x, X_{0}, X_{T}\right)}{\sqrt{T}}\right)^{2} \mathrm{~d} F_{S_{0}}(x) \\
& =\int_{-\infty}^{\infty}\left(\frac{1}{\sqrt{T}} \int_{0}^{T} \mathbb{I}_{\left\{X_{t} \leq x\right\}} \mathrm{d} W_{t}\right)^{2} \mathrm{~d} F_{S_{0}}(x)+O\left(\frac{1}{\sqrt{T}}\right),
\end{aligned}
$$

where we put

$$
H(x, y, z)=\int_{y}^{z} \frac{\mathbb{I}_{\{v \leq x\}}}{\sigma(v)} \mathrm{d} v .
$$

Note that the estimate $\mathbf{E}_{S_{0}} H\left(x, X_{0}, X_{T}\right)^{2}<C$ follows directly from the assumption $\mathcal{A}_{0}$ (see [1] for details). 
By the law of large numbers

$$
\frac{1}{T} \int_{0}^{T} \mathbb{1}_{\left\{X_{t} \leq x\right\}} \mathrm{d} t \longrightarrow F_{S_{0}}(x) .
$$

Hence, by the central limit theorem the stochastic integral

$$
\begin{aligned}
I_{T}(x) & =\frac{1}{\sqrt{T}} \int_{0}^{T} \mathbb{I}_{\left\{X_{t} \leq x\right\}} \mathrm{d} W_{t} \\
& =\frac{1}{\sqrt{T}} \int_{0}^{T} \mathbb{I}_{\left\{F_{S_{0}}\left(X_{t}\right) \leq F_{S_{0}}(x)\right\}} \mathrm{d} W_{t} \equiv J_{T}\left(F_{S_{0}}(x)\right)
\end{aligned}
$$

converges

$$
\left(I_{T}\left(x_{1}\right), \ldots, I_{T}\left(x_{k}\right)\right) \Longrightarrow\left(I\left(x_{1}\right), \ldots, I\left(x_{k}\right)\right)
$$

where

$$
I(x)=w\left(F_{S_{0}}(x)\right)
$$

Further, $\left(x_{1}<x_{2}\right)$

$$
\mathbf{E}_{S_{0}}\left|I_{T}\left(x_{2}\right)-I_{T}\left(x_{1}\right)\right|^{2}=F_{S_{0}}\left(x_{2}\right)-F_{S_{0}}\left(x_{1}\right) \leq C\left|x_{2}-x_{1}\right|
$$

because under coondition $\mathcal{A}_{0}$ the density $f_{S_{0}}(\cdot)$ is a bounded function.

The properties (15) and (16) yield the convergence

$$
\int_{-\infty}^{\infty}\left[I_{T}(x)\right]^{2} \mathrm{~d} F_{S_{0}}(x)=\int_{0}^{1}\left[J_{T}(t)\right]^{2} \mathrm{~d} t \Longrightarrow \int_{0}^{1} w(t)^{2} \mathrm{~d} t
$$

(Theorem A22, [9]). When we know the form of the statistic $\delta_{T}$ we can construct another goodness of fit test with the same asymptotic properties as follows. Let us introduce the statistic

$$
\delta_{T}^{*}=\int_{-\infty}^{\infty}\left(\frac{1}{\sqrt{T}} \int_{0}^{T} \frac{\mathbb{I}_{\left\{X_{t}<x\right\}}}{\sigma\left(X_{t}\right)}\left[\mathrm{d} X_{t}-S_{0}\left(X_{t}\right) \mathrm{d} t\right]\right)^{2} \mathrm{~d} F_{S_{0}}(x)
$$

and the constant $c_{\varepsilon}$ :

$$
\mathbf{P}\left\{\int_{0}^{1} w(t)^{2} \mathrm{~d} t>c_{\varepsilon}\right\}
$$

We assume that under the nonparametric alternative

$$
\mathcal{H}_{1} \quad: \quad \mathbf{E}_{S}\left(\frac{S(\xi)-S_{0}(\xi)}{\sigma(\xi)}\right)^{2}>0
$$

the function $S(\cdot)$ satisfies the conditions $\mathcal{E S}$ and $\mathcal{A}_{0}$. Here $\xi$ is the random variable with the density $f_{S}(x)$. Therefore the observed process is ergodic with the invariant density $f_{S}(\cdot)$.

Then for the test $\psi_{T}^{*}=\mathbb{I}_{\left\{\delta_{T}^{*}>c_{\varepsilon}\right\}}$ we have the following result. 
Proposition 1 The test $\psi_{T}^{*} \in \mathcal{K}_{\varepsilon}$ and is consistent against any fixed alternative $\mathcal{H}_{1}$.

Proof. Under hypothesis $\mathcal{H}_{0}$ we have

$$
\delta_{T}^{*}=\int_{-\infty}^{\infty}\left(\frac{1}{\sqrt{T}} \int_{0}^{T} \mathbb{I}_{\left\{X_{t}<x\right\}} \mathrm{d} W_{t}\right)^{2} \mathrm{~d} F_{S_{0}}(x)
$$

and it converges to the following limit

$$
\delta_{T}^{*} \Longrightarrow \int_{0}^{1} w(t)^{2} \mathrm{~d} t
$$

Therefore, the test $\psi_{T}^{*}=\mathbb{I}_{\left\{\delta_{T}^{*}>c_{\varepsilon}\right\}}$ belongs to $\mathcal{K}_{\varepsilon}$.

The consisteny follows from standard arguments as follows. Under alternative $\mathcal{H}_{1}$ we can write

$$
\begin{gathered}
\frac{1}{\sqrt{T}} \int_{0}^{T} \frac{\mathbb{I}_{\left\{X_{t}<x\right\}}}{\sigma\left(X_{t}\right)}\left[\mathrm{d} X_{t}-S_{0}\left(X_{t}\right) \mathrm{d} t\right]=\frac{1}{\sqrt{T}} \int_{0}^{T} \mathbb{1}_{\left\{X_{t}<x\right\}} \mathrm{d} W_{t} \\
+\frac{1}{\sqrt{T}} \int_{0}^{T} \frac{\mathbb{I}_{\left\{X_{t}<x\right\}}}{\sigma\left(X_{t}\right)}\left[S\left(X_{t}\right)-S_{0}\left(X_{t}\right)\right] \mathrm{d} t .
\end{gathered}
$$

The first (stochastic) integral is asymptotically normal

$$
I_{T}(x)=\frac{1}{\sqrt{T}} \int_{0}^{T} \mathbb{I}_{\left\{X_{t}<x\right\}} \mathrm{d} W_{t} \Longrightarrow w\left(F_{S}(x)\right) \sim \mathcal{N}\left(0, F_{S}(x)\right)
$$

and for the second we have by the law of large numbers

$$
M_{T}(x)=\frac{1}{T} \int_{0}^{T} \frac{\mathbb{I}_{\left\{X_{t}<x\right\}}}{\sigma\left(X_{t}\right)}\left[S\left(X_{t}\right)-S_{0}\left(X_{t}\right)\right] \mathrm{d} t \longrightarrow M(x) .
$$

Here

$$
M(x)=\mathbf{E}_{S}\left(\frac{\mathbb{I}_{\{\xi<x\}}}{\sigma(\xi)}\left[S(\xi)-S_{0}(\xi)\right]\right) .
$$

Denote

$$
\|h(\cdot)\|_{0}^{2}=\int_{-\infty}^{\infty} h(x)^{2} \mathrm{~d} F_{0}(x)
$$

Then we can write

$$
\begin{aligned}
\mathbf{P}_{S}\left(\delta_{T}^{*}>c_{\varepsilon}\right) & =\mathbf{P}_{S}\left(\left\|I_{T}(\cdot)+\sqrt{T} M_{T}(\cdot)\right\|_{0}>\sqrt{c_{\varepsilon}}\right) \\
& \geq \mathbf{P}_{S}\left(\sqrt{T}\left\|M_{T}(\cdot)\right\|_{0}-\left\|I_{T}(\cdot)\right\|_{0}>\sqrt{c_{\varepsilon}}\right) .
\end{aligned}
$$


Therefore, if $\left\|M_{T}(\cdot)\right\|_{0} \rightarrow\|M(\cdot)\|_{0}>0$ then the test is consistent.

The condition $\|M(\cdot)\|_{0}=0$ implies equality

$$
\int_{-\infty}^{x} \frac{\left[S(y)-S_{0}(y)\right]}{\sigma(y)} f_{S}(y) \mathrm{d} y=0 \quad \text { for } \quad \text { all } \quad x \in R .
$$

Hence

$$
\frac{S(x)-S_{0}(x)}{\sigma(x)} f_{S}(x)=0 \text { for almost all } x \in R,
$$

which is equivalent to the equality $S(x)=S_{0}(x)$ for almost all $x$. This contradicts the definition of the alternative.

We do not consider here the ADF test based on empirical distribution function, because the derivation of $\hat{\eta}_{T}(x)$ reduces to the test based on $\hat{\zeta}_{T}(x)$, for which we have already suggested a solution.

Remark. Note that the central statistic in (17) coincides with the statistic used in the Kolmogorov-Smirnov type test studied [15]. It is interesting to note that another Kolmogorov-Smirnov test based on empirical density $\zeta_{T}(x)=\sqrt{T}\left(\hat{f}_{T}(x)-f_{S_{0}}(x)\right)$ was studied in [11]. To compare these two tests we put $\sigma(x) \equiv 1$. Then the test in [15] is $\bar{\psi}_{T}\left(X^{T}\right)=\mathbb{I}_{\left\{\bar{\delta}_{T}>c_{\varepsilon}\right\}}$ with

$$
\bar{\delta}_{T}=\sup _{x} \frac{1}{\sqrt{T}}\left|\int_{0}^{T} \mathbb{I}_{\left\{X_{t}<x\right\}} \mathrm{d} X_{t}-\int_{0}^{T} \mathbb{I}_{\left\{X_{t}<x\right\}} S_{0}\left(X_{t}\right) \mathrm{d} t\right|
$$

It is shown that under hypothesis

$$
\bar{\delta}_{T} \Longrightarrow \sup _{0 \leq t \leq 1}|w(t)| .
$$

Therefore this test is ADF.

The test proposed in [11] is

$$
\delta_{T}^{\circ}=\sup _{x}\left|\zeta_{T}(x)\right|, \quad \psi_{T}^{\circ}\left(X^{T}\right)=\mathbb{I}_{\left\{\delta_{T}^{\circ}>c_{\varepsilon}\right\}} .
$$

It is shown that this statistic is asymptotically equivalent to the statistic

$$
\delta_{T}^{+}=\sup _{x} \frac{2}{\sqrt{T}}\left|\int_{0}^{T} \mathbb{I}_{\left\{X_{t}<x\right\}} \mathrm{d} X_{t}-\mathbf{E}_{S_{0}} \int_{0}^{T} \mathbb{I}_{\left\{X_{t}<x\right\}} S_{0}\left(X_{t}\right) \mathrm{d} t\right|
$$

and it converges to the limit

$$
\delta_{T}^{+} \Longrightarrow \sup _{x}|\Phi(x)| f_{0}(x) .
$$

The comparison of (18) and (19) shows the advantage of (18) because it is $\mathrm{ADF}$. 


\section{References}

[1] Choulakian, V. and Stephens, M.A. (2001), Goodness-of-fit tests for the generalized Pareto distribution. Technometrics, 43, 478-484.

[2] Dachian, S. and Kutoyants, Yu.A. (2003) On cusp estimation for ergodic diffusion. J. Stat. Planning Infer. , 117, 153-166.

[3] Dachian, S. and Kutoyants, Yu.A. (2007) On the goodness-of-fit tests for some continuous time processes, in Statistical Models and Methods for Biomedical and Technical Systems, F.Vonta et al. (Eds), Birkhäuser, Boston, 395-413.

[4] Darling, D. A. (1955) The Cramér-Smirnov test in the parametric case. Ann. Math. Statist., 26, 1-20.

[5] Durbin, J. (1973) Weak convergence of the sample distribution function when parameters are estimated. Ann. Statist., 1, 2, 279-290.

[6] Durett, R. (1996) Stochastic Calculus. A practical introduction. Boca Raton: CRC Press.

[7] Fournie, E. (1992) Un test de type Kolmogorov-Smirnov pour processus de diffusions ergodic. Rapport de Recherche, 1696, INRIA, SophiaAntipolis.

[8] Fournie, E., Kutoyants, Yu. A. (1993) Estimateur de la distance minimale pour des processus de diffusion ergodiques. Rapport de Recherche, 1952, INRIA, Sophia-Antipolis.

[9] Ibragimov, I.A. and Khasminskii, R.Z. (1981). Statistical Estimation. Springer, New York.

[10] Kac, M., Kiefer, J. and Wolfowitz, J. (1955) On tests of normality and other tests of goodness of fit based on distance methodes.Ann. Mathem. Statist., 26, 189-211.

[11] Kutoyants, Yu.A. (2004) Statistical Inference for Ergodic Diffusion Processes, Springer, London.

[12] Kutoyants Yu. A., (2010) On the goodness-of-fit testing for ergodic diffusion processes. J. Nonparamet. Statist. 22, 4, 529-543.

[13] Lehmann, E.L. and Romano, J.P. (2005) Testing Statistical Hypotheses. (3rd ed.) Springer, N.Y. 
[14] Martynov, G. (2010) Note on Cramér-von Mises test with estimated parameters. Publ. Math. Debrecen, 76/3, 341-346.

[15] Negri, I. and Nishiyama, Y. (2009) Goodness of fit test for ergodic diffusion processes. Ann. Inst. Statist. Math., 61, 919-928.

[16] Negri, I., and Zhou, L. (2012) On goodness-of-fit testing for ergodic diffusion process with shift parameter, arXiv:1203.6547, sumitted.

[17] Revuz, D. and Yor, M. (1991) Continuous Martingales and Brownian Motion. Springer, N.Y. 